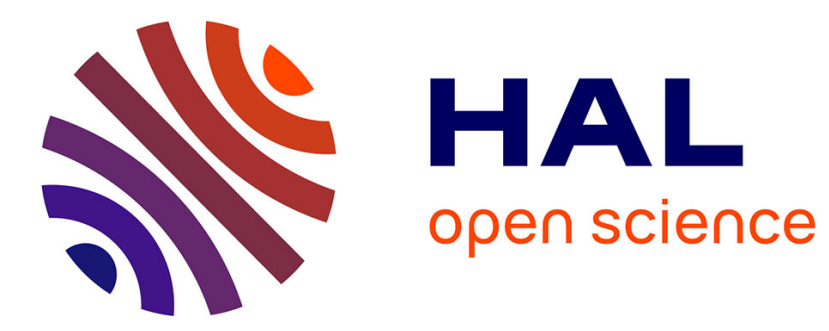

\title{
Relativistic mass due to a dilatant vacuum leads to a quantum reformulation of the relativistic kinetic energy
}

Marco Fedi

\section{To cite this version:}

Marco Fedi. Relativistic mass due to a dilatant vacuum leads to a quantum reformulation of the relativistic kinetic energy. 2019. hal-01898011v4

\section{HAL Id: hal-01898011 \\ https://hal.science/hal-01898011v4}

Preprint submitted on 19 Apr 2019

HAL is a multi-disciplinary open access archive for the deposit and dissemination of scientific research documents, whether they are published or not. The documents may come from teaching and research institutions in France or abroad, or from public or private research centers.
L'archive ouverte pluridisciplinaire HAL, est destinée au dépôt et à la diffusion de documents scientifiques de niveau recherche, publiés ou non, émanant des établissements d'enseignement et de recherche français ou étrangers, des laboratoires publics ou privés. 


\title{
Relativistic mass due to a dilatant vacuum leads to a quantum reformulation of the relativistic kinetic energy
}

\author{
Marco Fedi ${ }^{*}$ \\ Ministero dell'Istruzione, dell'Università e della Ricerca (MIUR), Rome, Italy
}

\begin{abstract}
Relativistic mass change with speed is considered as the effect of a viscous, dilatant vacuum, whose apparent viscosity is related to the Lorentz factor. Transient solidlike vacuum due to shear stress would be the reason why vacuum prevents the speed of massive objects from being indefinitely increased. Such a vacuum (that in a previous study allowed to exactly calculate the Pioneer anomaly, Mercury's perihelion precession and was shown to be compatible with stable planetary orbits) leads us here to a quantum formula for the relativistic kinetic energy. A formula which distinguishes between the case of accelerated charges in a vacuum, for which a Stokes-Einstein radius comes into play, and the case of accelerated macroscopic bodies, for which the quantum potential term vanishes. In this way, incidentally, one obtains again correct results for the Pioneer 10, confirming the role of vacuum's viscous force. This description of a quantum mechanism underlying the relativistic kinetic energy may be also helpful in constructing a theory of quantum relativity and might tell us more even about the interactions of matter with the Higgs field and the dark sector: two issues which can be themselves linked to a dilatant vacuum.
\end{abstract}

PACS numbers: 95.30.Lz; 03.30.+p; 83.60.Rs; 41.75.-i

Keywords: dilatant vacuum; relativistic kinetic energy; Stokes-Einstein radius in a vacuum; quantum relativity

\section{INTRODUCTION}

In particle acceleration, there is an enormous discrepancy between the energy supplied to a particle and its resulting speed, which is much lower than expected, according to classical physics. Accelerated particles seem to meet an impenetrable wall, located at the speed of light: the greatest part of the supplied energy seems to get lost, besides a fraction of it, converted into synchrotron light. According to special relativity theory (SRT), this gap is due to energy which converts into relativistic mass. This concept is, however, still rather controversial [2, 3]. It is actually preferred to refer to relativistic kinetic energy, instead of relativistic mass, according to the well-known formula $E_{k}=$ $m c^{2}\left(\left(1 / \sqrt{1-(v / c)^{2}}\right)-1\right)$, but in any case, mass is the only parameter of this equation that can take charge of the supplied energy which is not converted into classical kinetic energy. At least if physical vacuum, the Higgs field or the dark sector are not taken into account and the equation is not modified into a quantum equation. In the light of modern physics, we could for instance hypothesize that massive bodies which are accelerated through the Higgs field are subject to its apparent viscosity, which would obey the Lorentz factor, acquiring further mass. This situation would mean that the Higgs field is a dilatant fluid and that it also interacts with macroscopic, massive bodies. Higgs field or simply a viscous vacuum. This is not only a hypothesis, since a modified Stokes' law for a dilatant vacuum [1], in which the Lorentz factor has the function of a dimensionless viscosity term (in place of $\eta$ in the classical Stokes' law), produced direct and exact solutions to the Pioneer anomaly (replacing the more complicate and approximate thermal simulations, previously proposed as a solution to that anomaly [1]) and to Mercury's perihelion

\footnotetext{
*E-mail: marco.fedi@istruzione.it
}

precession, i.e. to the first classical test of general relativity. Section III recalls then the modified Stokes' equation for a dilatant vacuum, while Sect III treats proton acceleration in a dilatant vacuum and allows to achieve a quantum formula for relativistic kinetic energy. Pointing out that relativistic mass is actually a detected greater inertia (greater difficulty to accelerate a body) due not to an increasing relativistic mass but to a dilatant vacuum which obeys the Lorentz factor $(\gamma-1$, precisely), the role of rest mass in the interaction with the vacuum is differently presented in Sect. IV via 3D plots of the Lorentz factor, which show that the factor tends to a right angle, depending on mass. In the subsequent section (V), calculations are presented as regards the acceleration of macroscopic bodies.

\section{REINTERPRETING RELATIVISTIC MASS VIA VACUUM DILATANCY}

A modified Stokes' equation (MSE), obtained by replacing in Stokes' law the viscosity coefficient multiplied by speed, $\eta \nu$, with $(\gamma-1) \kappa$, where $\gamma$ is the Lorentz factor, here reinterpreted as a nonlinear, shear-depending term of vacuum dilatancy, has been able to exactly solve two famous anomalies [1] and should be therefore considered as an evidence that physical vacuum is a shear-thickening fluid. The MSE, expressing vacuum's viscous force, reads

$$
\begin{aligned}
F_{\emptyset} & =-6 \pi r\left(\frac{1}{\sqrt{1-\left(\frac{v}{c}\right)^{2}}}-1\right) \kappa=-6 \pi r(\gamma-1) \kappa \\
& =-6 \pi r D \kappa
\end{aligned}
$$

where $\gamma$ is the Lorentz factor, $\kappa$ is a unitary constant expressed in $\mathrm{kg} / \mathrm{s}^{2}$, the subscript $\emptyset$ refers to the vacuum and

$$
D=\gamma-1
$$


is the term of vacuum dilatancy, whose asymptote means that a solidlike state of the vacuum due to sufficiently large shear stress can be reached and an indefinitely high viscosity comes into play, impeding further acceleration. Taking into account the existence of a dilatant vacuum, it follows that relativistic kinetic energy is the additional energy to be provided to a body to oppose the dilatant vacuum: Which results in an increased inertia of the body. Indeed, Einstein equation for relativistic kinetic energy is actually nothing but the rest energy of a body multiplied by the term of vacuum dilatancy (2)

$$
E_{k}=m c^{2} D \text {. }
$$

Section III describes this aspect (and a quantum formula is therein obtained).

Dilatant vacuum is therefore suggested as a step towards a quantum theory of relativity. Indeed, a previous positive test on the equation of general relativity expressing Mercury's perihelion precession was done in [1]. The opposite force (a drag force) exerted by physical vacuum is detected in synchrotrons as increased inertia, and consequently thought of as increased (relativistic) mass, but a better and quantum-oriented explanation is suggested below, in terms of the reaction of a shear-thickening vacuum, as the calculations in Sect. V]confirm.

\section{PARTICLE ACCELERATION IN DILATANT VACUUM}

Let us consider the case of a proton accelerated up to $E_{k}=6.5 \mathrm{TeV}=1.04141 \times 10^{-6} \mathrm{~J}$. According to classical physics it would have the following speed $\left(v_{c}\right)$

$$
\begin{aligned}
v_{c} & =\sqrt{\frac{2 E_{k}}{m}}=\sqrt{\frac{2 \cdot\left(1.04141 \times 10^{-6} \mathrm{~J}\right)}{1.67262 \times 10^{-27} \mathrm{~kg}}}= \\
& =3.5288 \times 10^{10} \mathrm{~m} \cdot \mathrm{s}^{-1},
\end{aligned}
$$

where $m$ refers here and below to rest mass, but in particle accelerators a much lower speed (observed speed, $v_{o}$ ) is detected. In this case

$$
\begin{aligned}
& v_{o}= c \sqrt{1-\left(\frac{E_{k}}{m c^{2}}+1\right)^{-2}}=\left(299792458 \mathrm{~m} \cdot \mathrm{s}^{-1}\right) \cdot \\
& \cdot \sqrt{1-\left(\frac{\left(1.04141 \times 10^{-6} \mathrm{~J}\right)}{\left(1.67262 \times 10^{-27} \mathrm{~kg}\right)\left(299792458 \mathrm{~m} \cdot \mathrm{s}^{-1}\right)^{2}}+1\right)^{-2}} \\
&=299792454.8775 \mathrm{~m} \cdot \mathrm{s}^{-1}
\end{aligned}
$$

and this fact is explained as relativistic kinetic energy. That could be also explained via the Higgs field. Indeed that field is like a viscous fluid which impedes particles and gives them mass. As for classical viscous fluids, we could hypothesize that acceleration through the Higgs field causes increasing apparent viscosity: that would result in the detection of increased inertia for accelerated particles. The Higgs field would not only give each particle its specific rest mass but it would also be the quantum cause of the phenomenon called relativistic mass. In this study, however, the more general concept of shear-thickening vacuum impeding accelerated particles is used, and the possibility that this kind of vacuum coincides with the Higgs field is suggested and left to subsequent further investigation and tests. But also considering the vacuum as a doped superfluid (e.g. dark matter particles diffused in a sea of superfluid dark energy) might anyway account for vacuum dilatancy. It is furthermore interesting to notice that the $T^{00}$ component of the stress-energy tensor in Einstein field equation may refer to both vacuum's mass density and relativistic mass per unit volume. Furthermore, the $T^{21}, T^{31}, T^{32}$ components refer to momentum flux, which is related to viscosity (as momentum diffusion due to shear stress) via Newton's law for viscosity, while $T^{12}, T^{13}, T^{23}$ directly refer to shear stress. In short, Higgs field, dark sector, Einstein field equation and dilatant vacuum are closely related and they point in the same direction [4]: a viscous, shear-thickening vacuum, whose apparent viscosity obeys the Lorentz factor.

In the example of a proton at $6.5 \mathrm{TeV}$, vacuum friction should cause the following speed decrease

$$
\Delta v=v_{o}-v_{c}=-3.49882 \times 10^{10} \mathrm{~m} \cdot \mathrm{s}^{-1} .
$$

This can be also written $\Delta v=a_{\emptyset} \Delta t$, where $\Delta t=1 \mathrm{~s}$ and

$$
a_{\emptyset}=\frac{F_{\emptyset}}{m}=\frac{-6 \pi r_{h(\emptyset)} D \kappa}{m}=\frac{-6 \pi\left(4.48164 \times 10^{-22} \mathrm{~m}\right)\left(\frac{1}{\left.\sqrt{1-\left(\frac{299792454.8775 \mathrm{~m} \cdot \mathrm{s}^{-1}}{299792458 \mathrm{~m} \cdot \mathrm{s}^{-1}}\right)^{2}}-1\right) \cdot 1 \mathrm{~kg} \cdot \mathrm{s}^{-2}}\right.}{1.67262 \times 10^{-27} \mathrm{~kg}}=-3.49882 \times 10^{10} \mathrm{~m} \cdot \mathrm{s}^{-2}
$$

is the negative acceleration caused by dilatant vacuum. where $r_{h(\emptyset)}$ is the hydrodynamic Stokes-Einstein radius (being physical vacuum the fluid at issue). The use of proton charge radius in this hydrodynamic context would be indeed nonsensical. The interaction proton-vacuum can be due to electric interactions with the virtual dipoles in the vacuum (particle-antiparticle pairs). Here is the derivation of the hydrodynamic radius for particles moving in a fluid, dilatant vacuum: from Stokes-Einstein formula $r_{h}=k_{B} T / 6 \pi \eta d$, where $k_{B}$ is the Boltzmann constant, $T$ is temperature, $\eta$ the viscous coefficient of the classical Stokes' law and $d$ the diffusion coefficient, since $k_{B} T=p V / N$, where 
$p, V, N$ are pressure, volume and the number of vacuum's quanta (respectively), the hydrodynamic radius reads $r_{h}=$ $p V / N 6 \pi \eta d$. Moreover, $d=\mu k_{B} T$, where $\mu$ is the mobility coefficient (in this case of vacuum's quanta), so we can write the following rightmost equivalence $\mu=v_{d} p V / F N=$ $v_{o} p V / F_{\emptyset} N$, where $v_{d}=v_{o}$ (with $v_{d}$ being the terminal drift velocity) and the applied force $(F)$ is vacuum's viscous force $\left(F_{\emptyset}\right.$, the MSE), according to Newton's third law (vacuum's reaction). The hydrodynamic radius becomes then $r_{h}=$ $F_{\emptyset} / 6 \pi \eta v_{o}$. Finally, since to derive the MSE, the substitution $\eta v=D \kappa$ was done (to describe a dilatant vacuum), one arrives to the equation $r_{h}=m a_{\emptyset} /(-6 \pi D \kappa)$, where $a_{\emptyset}$ is negative, so the radius has a positive value. After some algebra, one can proceed with the following equivalences

$$
r_{H(\emptyset)}=-\frac{m a_{\emptyset}}{6 \pi D \kappa}=-\frac{m \Delta v}{6 \pi D \kappa \Delta t}=-\frac{m^{2} c^{2} \Delta v}{6 \pi E_{k} \kappa \Delta t}
$$

where the term of vacuum dilatancy (2), $D$, contains $v_{o}$ and $\Delta v=v_{o}-v_{c}$, as above.

Eq. (6) can be also expressed in terms of $\Delta E_{k}$, that is

$$
W_{\emptyset} \equiv \Delta E_{k}=\frac{1}{2} m\left(v_{o}^{2}-v_{c}^{2}\right)
$$

and since

$$
v_{o}^{2}-v_{c}^{2}=(\Delta v)^{2}+2 v_{o} v_{c}-2 v_{c}^{2}
$$

we obtain the work done by the dilatant vacuum to brake the proton, also observable (with opposite sign) as the energy spent by the synchrotron to oppose vacuum dilatancy

$$
\begin{aligned}
& W_{\emptyset}=\Delta E_{k}= \\
= & \frac{1}{2} m\left[(\Delta v)^{2}+2 v_{o} v_{c}-2 v_{c}^{2}\right]= \\
= & \frac{1}{2} m\left[(\Delta v)^{2}+2 v_{c} \Delta v\right]=\frac{1}{2} m\left[\left(a_{\emptyset} \Delta t\right)^{2}+2 v_{c} a_{\emptyset} \Delta t\right]= \\
= & a_{\emptyset} \Delta t\left(\frac{1}{2} m a_{\emptyset} \Delta t+m v_{c}\right)=\frac{F_{\emptyset} \Delta t}{m}\left(\frac{F_{\emptyset} \Delta t}{2}+m v_{c}\right)= \\
= & \frac{\left(F_{\emptyset} \Delta t\right)^{2}}{2 m}+F_{\emptyset} \Delta t v_{c}=\frac{\left(\int F_{\emptyset} d t\right)^{2}}{2 m}+v_{c} \int F_{\emptyset} d t=\frac{J_{\emptyset}^{2}}{2 m}+J_{\emptyset} v_{c},
\end{aligned}
$$

where $J_{\emptyset}$ is the impulse due to vacuum's viscous force. The negative work of the vacuum on the accelerated proton is

$$
\begin{aligned}
W_{\emptyset}=\Delta E_{k}=\frac{J_{\emptyset}^{2}}{2 m}+J_{\emptyset} v_{c}=\left(-6 \pi\left(4.48164 \times 10^{-22} \mathrm{~m}\right)\left(\frac{1}{\sqrt{1-\left(\frac{299792454.8775 \mathrm{~m} \cdot \mathrm{s}^{-1}}{299792458 \mathrm{~m} \cdot \mathrm{s}^{-1}}\right)^{2}}-1}\right) \cdot 1 \mathrm{~kg} \cdot \mathrm{s}^{-2} \cdot 1 \mathrm{~s}\right)^{2} \cdot \\
\quad \cdot \frac{1}{2 \cdot\left(1.67262 \times 10^{-27} \mathrm{~kg}\right)}+\left(-6 \pi\left(4.48164 \times 10^{-22} \mathrm{~m}\right)\left(\frac{1}{\left.\sqrt{1-\left(\frac{299792454.8775 \mathrm{~m} \cdot \mathrm{s}^{-1}}{299792458 \mathrm{~m} \cdot \mathrm{s}^{-1}}\right)^{2}}-1\right) \cdot 1 \mathrm{~kg} \cdot \mathrm{s}}\right) \cdot 1 \mathrm{~s}\right) \cdot \\
\quad \cdot\left(3.5288 \times 10^{10} \mathrm{~m} \cdot \mathrm{s}^{-2}\right)= \\
=-1.04133 \times 10^{-6} \mathrm{~J}
\end{aligned}
$$

part of which converted into synchrotron radiation: for the case of protons just a tiny amount. The corresponding equation for relativistic kinetic energy (total supplied energy) reads then

$$
E_{k}=-W_{\emptyset}+E_{k}^{(\text {class })}
$$

where $E_{k}^{(\text {class })}=m v_{o}^{2} / 2$ is the classical kinetic energy. Thus

$$
\begin{aligned}
E_{k} & =m c^{2}\left(\frac{1}{\sqrt{1-\left(\frac{v_{o}}{c}\right)^{2}}}-1\right)= \\
& =-\left(\frac{J_{\emptyset}^{2}}{2 m}+J_{\emptyset} v_{c}\right)+\frac{1}{2} m v_{o}^{2} .
\end{aligned}
$$

where the minus sign allows us to observe the total energy amount supplied to the proton. In fact, Eq. (14) yields the correct energy corresponding to $6.5 \mathrm{TeV}$. This energy amount can be interpreted as the classical kinetic energy the proton has at $v_{o}$, considering its rest mass, plus the work done by the accelerator on the particle to accelerate it against the dilatant vacuum and equal, for the third principle of motion, to the negative work of the vacuum, splitted into two components, one of which is revealed below as a quantum potential. Let us express Eq. 11 - with minus sign to refer to the supplied energy, which is specular with respect to the negative work of the vacuum - by resorting to momentum $(p)$,

$$
-\left(\frac{J_{\emptyset}^{2}}{2 m}+J_{\emptyset} v_{c}\right)=\frac{(\Delta p)^{2}}{2 m}+\Delta p v_{c} .
$$

Let us define the momentum values $p_{1}=J_{\emptyset}$ and $p_{2}=m v_{c}$. The right member of Eq. 15 can be written

$$
\frac{p_{1}^{2}}{2 m}\left(1+\frac{2 p_{2}}{p_{1}}\right)
$$

In the momentum basis, switching to the momentum operator, $p=\hat{p}$, we obtain from Eq. (16) 


$$
\begin{aligned}
& \frac{p_{1}^{2}}{2 m}\left(1+\frac{2 p_{2}}{p_{1}}\right) \Rightarrow\left[\frac{\hat{p}_{1}^{2}}{2 m}\left(1+\frac{2 \hat{p}_{2}}{\hat{p}_{1}}\right)\right] \Psi(\mathbf{r}, t)= \\
& =\left[-\frac{\hbar^{2}}{2 m} \nabla_{1}^{2}\left(1+\frac{2 \nabla_{2}}{\nabla_{1}}\right)\right] \Psi(\mathbf{r}, t)= \\
& =\hat{T} \Psi(\mathbf{r}, t) \stackrel{\hat{V} \Psi(\mathbf{r}, t)=0}{\Longrightarrow}=\hat{H} \Psi(\mathbf{r}, t)=i \hbar \frac{\partial \Psi(\mathbf{r}, t)}{\partial t}
\end{aligned}
$$

where $\psi$ is the wave function for the proton and the potential is zero, $\hat{V} \Psi(\mathbf{r}, t)=0$ (free particle). Eq. 17) therefore represents the quantum equation expressing the work of the vacuum on the accelerated particle and also the corresponding energy spent by the accelerator to oppose vacuum dilatancy. In Eq. [11, kinetic energy is expressed as the sum of two components and this fact will be important in Sect. V. analyzing the acceleration of massive, macroscopic bodies, a case in which the second addend vanishes and Eq. (14) produces correct results unlike SRT. The equations above, which resort to momentum for treating relativistic kinetic energy, also agree with Okun [3], who points out that referring to momentum to describe relativistic mass is more desirable. From Eq. [16] the expectation value for the kinetic energy is

$$
\begin{aligned}
\langle E\rangle & =\frac{\left\langle p_{1}^{2}\right\rangle}{2 m}\left(1+\frac{2\left\langle p_{2}\right\rangle}{\left\langle p_{1}\right\rangle}\right)= \\
& =\int_{-\infty}^{\infty} \Psi^{*}(\mathbf{r}, t)\left[-\frac{\hbar^{2}}{2 m} \nabla_{1}^{2}\left(1+\frac{2 \nabla_{2}}{\nabla_{1}}\right)\right] \Psi(\mathbf{r}, t) d^{3} \mathbf{r} .
\end{aligned}
$$

As we know, the imaginary part of the Schrödinger equation in polar form, putting the amplitude squared equal to the probability density $\Psi^{*} \Psi=R^{2}=\rho$ yields the continuity equation

$$
\frac{\partial \rho}{\partial t}+\nabla \cdot\left(\frac{\rho \nabla S}{m}\right)=0
$$

which can here represent the sum of the observed kinetic energy variation and the energy transferred to the dilatant vacuum, until a collision between particles occurs $\left(\nabla \cdot\left(\frac{\rho \nabla S}{m}\right)=\nabla \cdot \mathbf{j}\right.$, where $\mathbf{j}$ is the probability current, here interpreted as energy flux), due to viscosity being momentum transfer (that implies a $\Delta E_{k}$ ), and this gives the total, conserved, energy supplied to the proton by the accelerator. In this case, energy conservation occurs via the exchange with the vacuum. The detected increased inertia of the accelerated proton is currently interpreted as relativistic mass or, in the Higgs picture, one can say that the apparent viscosity of the Higgs field, due to acceleration through the field, implies a further mass acquisition process, interpretable as relativistic mass, but actually still in form of increased inertia, due to the opposition of the vacuum (of the Higgs field). If we prefer to involve the Higgs field, it has then to be treated as a viscous, dilatant fluid. In this case, the work of the shear-thickening vacuum on the accelerated proton is that of the Higgs field $(\phi)$, i.e. $W_{\emptyset}=W_{\phi}$.

The real part of the Schrödinger equation in polar form yields

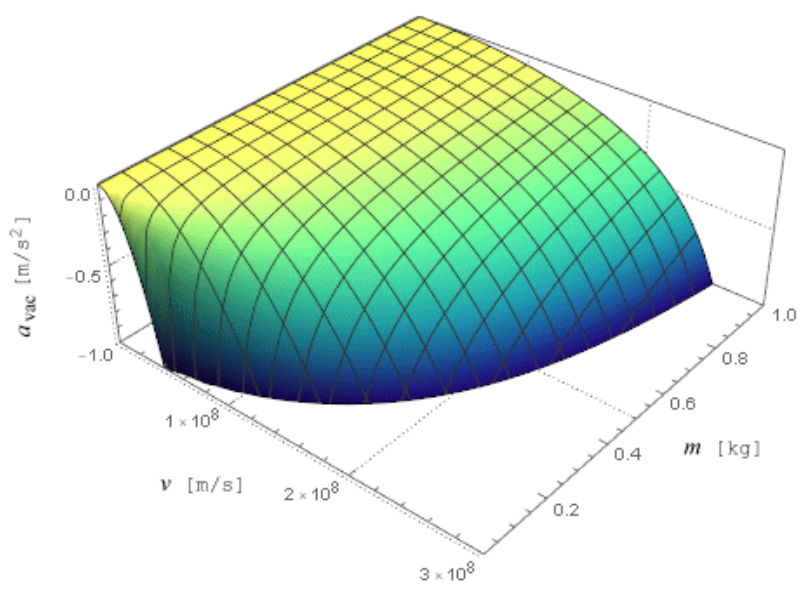

FIG. 1. Plot of $a_{\emptyset}=F_{\emptyset} / m$, in which $F_{\emptyset}$ contains the Lorentz factor in the form $\gamma-1$ as a term of vacuum dilatancy. The greater the mass the lower the negative acceleration caused by dilatant vacuum.

then the quantum Hamilton-Jacobi equation

$$
\frac{\partial S}{\partial t}=-\frac{|\nabla S|^{2}}{2 m}+Q
$$

where, in this case, potential energy is zero. Here $S$ can be the action (units are $\mathrm{J} \cdot \mathrm{s}$ ) of dilatant vacuum on the accelerated proton, i.e. the work done on the proton, which settles on $v_{o}$. However, also the quantum potential

$$
Q=-\frac{\hbar^{2}}{2 m} \frac{\nabla^{2} \sqrt{\rho}}{\sqrt{\rho}}
$$

is expressed as kinetic energy and has an analogous meaning. From Eq. (15), we deduce it refers to that part of $W_{\emptyset}$ corresponding to $p v_{c}=p_{1} p_{2} / m$. In Sect. $\mathrm{V}$, we will see that this quantum potential vanishes for macroscopic bodies, for which the hydrodynamic radius is not used.

\section{LORENTZ FACTOR 3D: ROLE OF MASS}

In the present framework relativistic mass has vanished, replaced by the viscous force of a dilatant vacuum, and a different role of mass emerges. Indeed, from Newton's second law, we see that the greater the mass, the weaker the acceleration: $a=F / m$. That is valid also for the negative acceleration caused by shear-thickening vacuum. This fact means that bodies with a greater mass require of course more energy to be accelerated but they are less slowed down by the dilatant vacuum (Fig. 1). The greater the mass the lower the kinetic energy dissipation caused by dilatant vacuum. The fact that a larger mass implies lower kinetic energy dissipation (in this picture let us say lower deceleration caused by dilatant vacuum) is after all known in particle acceleration, when comparing electrons with protons: being the latters about 1836 times heavier than electrons, the dissipation of kinetic energy is much smaller. Indeed, the radiated power

$$
P=\frac{e^{4}}{6 \pi \varepsilon_{0} c^{5}} \frac{B^{2}}{m^{4}}\left(E^{2}-m^{2} c^{4}\right)
$$



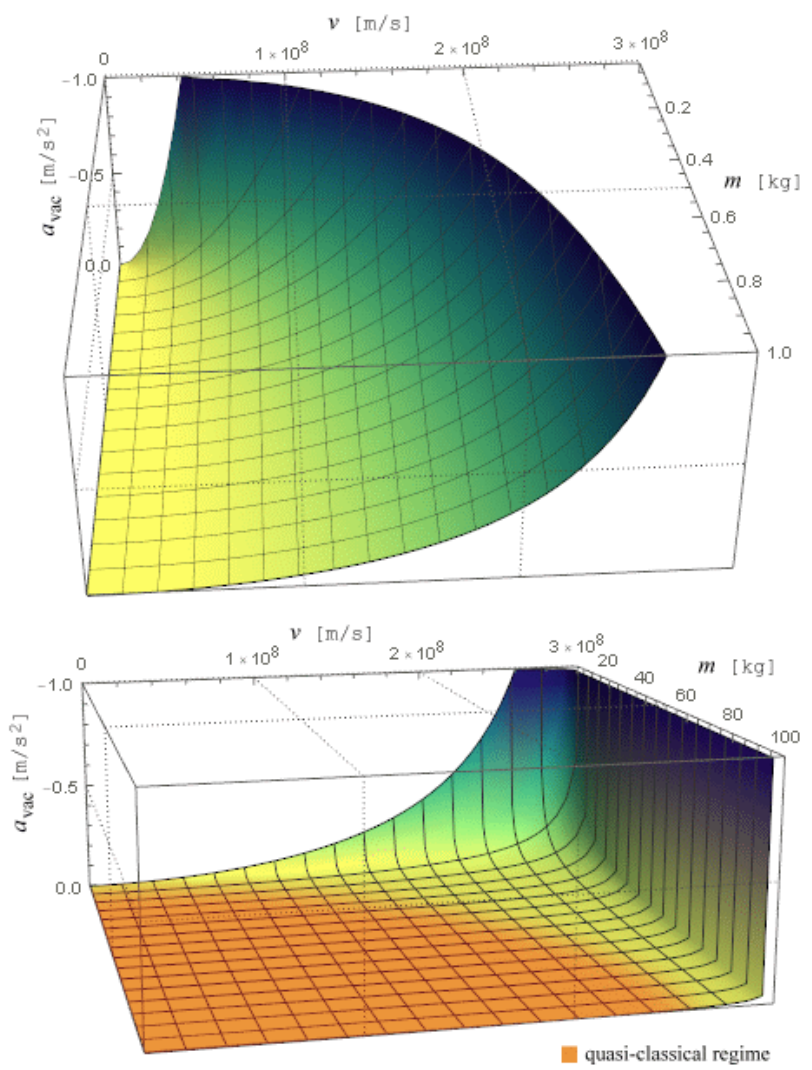

FIG. 2. Two graphs showing the negative acceleration provoked by dilatant vacuum as speed and mass vary. Above, a light mass encounters soon great deceleration, while one kilogram mass suffers a much lower negative acceleration. Below, as mass increases, a body can reach quasi-luminal speed without suffering the relativistic effects of special relativity, i.e. without being appreciably decelerated by the physical vacuum. We see that the Lorentz factor-driven curve tends, indeed, to a right angle by accelerating larger masses. Quasi-classical regime extends then depending on mass.

depends, inter alia, on the reciprocal of the mass (rised to the fourth). So, the smaller mass of electrons causes greater kinetic energy dissipation (as synchrotron radiation) and here this fact is explained via a dilatant vacuum: $a_{\emptyset}=F_{\emptyset} / m$, where again the reciprocal of the mass appears. As shown in Fig. 2, as a larger rest mass is considered, the Lorentz factor progressively bends at a right angle. More massive bodies can be therefore accelerated toward the speed of light remaining longer in a quasi-classical regime. That indicates an enormous difference, currently ignored, between the world of subatomic particles and that of macroscopic bodies, as far as the relativistic kinetic energy is concerned. By applying $a_{\emptyset}=F_{\emptyset} / m$, we have seen (7) that a proton close to the speed of light is decelerated in the order of magnitude of $-10^{10} \mathrm{~m} \cdot \mathrm{s}^{-2}$, while if the International Space Station, whose mass is 419 tons, were accelerated up to $0.99 c$, it would suffer a deceleration of only $0.001 \mathrm{~m} \cdot \mathrm{s}^{-2}$. Thus, missions based on ultra-light devices [8] would fail under the enormous deceleration caused by dilatant vacuum. If solidified vacuum is not a rigid body, the collapse of its lattice under a sufficiently large accelerated mass would remove every obstacle to indefinitely exceeding the speed of light. The vertical asymptote at the speed of light is in fact explained in this framework as a transient, shear-driven, quasi-lattice formation in the vacuum, which therefore acts as an impenetrable wall.

\section{ACCELERATION OF MACROSCOPIC BODIES: DILATANT VACUUM VS. SPECIAL RELATIVITY}

Let us see now how the new equation for kinetic energy (14) behaves as regards the acceleration of macroscopic bodies, for which the hydrodynamic Stokes-Einstein radius cannot be taken into account. Let us consider the famous Pioneer anomaly, which was declared solved in 2012 [5]. The suggested thermal simulations which claim to have explained that anomaly are very approximate and based on several uncertainties [6] while Eq. (14) gives a direct and exact result [1], demonstrating that the Pioneer anomaly has been caused by a dilatant vacuum. It is important to distinguish between the case of accelerated ions in quantum vacuum (23), as the case of the proton above, for which the Stokes-Einstein radius must be used, and the case of macroscopic objects (24), for which the normal geometrical radius is used and the term referring to quantum potential 20] vanishes

$$
\begin{gathered}
W_{\emptyset}^{(q)}=\frac{J_{\emptyset}^{2}}{2 m}+J_{\emptyset} v_{c} \\
W_{\emptyset}^{(M)}=-\frac{J_{\emptyset}^{2}}{2 m},
\end{gathered}
$$

where the superscripts $(q)$ and $(M)$ refer to charged particles and macroscopic bodies (respectively). Whereas the equation corresponding to Einstein formula for relativistic kinetic energy, which makes no distinction between charged particles and macroscopic bodies as the interaction with the dilatant vacuum is concerned, appears in (14). In the case of the NASA spacecraft Pioneer 10, the quantum-potential-related term vanishes and the negative work of the dilatant vacuum on the spacecraft, as subtracted kinetic energy, reduces to (24)

$$
\begin{aligned}
& W_{\emptyset}^{(M)}=-\frac{J_{\emptyset}^{2}}{2 m}= \\
& =-\left(-6 \pi(1.371 \mathrm{~m})\left(\frac{1}{\sqrt{1-\left(\frac{36737 \mathrm{~m} \cdot \mathrm{s}^{-1}}{299792458 \mathrm{~m} \cdot \mathrm{s}^{-1}}\right)^{2}}}-1\right) \cdot 1 \mathrm{~s}\right)^{2} . \\
& \cdot \frac{1}{2 \cdot 222 \mathrm{~kg}}=-8.479 \times 10^{-17} \mathrm{~J}
\end{aligned}
$$

where $36737 \mathrm{~m} / \mathrm{s}$ is the maximum speed of the probe after the Jupiter flyby [7] and $222 \mathrm{~kg}$ is the probe mass (i.e. 258 $\mathrm{kg}$ minus $36 \mathrm{~kg}$ burned hydrazine). In fact, by applying the classical equation $a=\Delta v / \Delta t$, with the energy value obtained in Eq. (25), we see that the acceleration exactly corresponds 
to that detected by the NASA

$$
\begin{aligned}
a_{P} & =-\frac{1}{\Delta t} \sqrt{\frac{2\left|W_{\emptyset}\right|}{m}}=-\frac{1}{1 \mathrm{~s}} \sqrt{\frac{2 \cdot\left(8.479 \times 10^{-17} \mathrm{~J}\right)}{222 \mathrm{~kg}}}= \\
& =-8.74 \times 10^{-10} \mathrm{~m} \cdot \mathrm{s}^{-2}
\end{aligned}
$$

(see also [1]) where the minus sign compensates for the necessary modulus under root, to obtain negative acceleration. On the contrary, the formula of special relativity for relativistic kinetic energy, $E_{k}^{(r e l)}$, once the classical kinetic energy $\left(E_{k}^{(\text {class })}=m v_{o}^{2} / 2\right.$, with $m$ rest mass $)$ is subtracted, yields the following energy value

$$
\begin{aligned}
E_{k}^{(\text {rel })}-E_{k}^{(\text {class })} & =m c^{2}\left(\frac{1}{\sqrt{1-\left(\frac{v}{c}\right)^{2}}}-1\right)-\frac{1}{2} m v_{o}^{2}= \\
& =(222 \mathrm{~kg})\left(299792458 \mathrm{~m} \cdot \mathrm{s}^{-1}\right)^{2} \cdot \\
& \cdot\left(\frac{1}{\sqrt{1-\left(\frac{36737 \mathrm{~m} \cdot \mathrm{s}^{-1}}{299792458 \mathrm{~m} \cdot \mathrm{s}^{-1}}\right)^{2}}}-1\right)- \\
& -\frac{1}{2}(222 \mathrm{~kg})\left(36737 \mathrm{~m} \cdot \mathrm{s}^{-1}\right)^{2}= \\
& =1687 \mathrm{~J}
\end{aligned}
$$

which does not account for the anomalous variation of kinetic energy of the Pioneer 10, and this value would imply an anomalous $\Delta v$ of $-3.89 \mathrm{~m} / \mathrm{s}$. NASA should at this point absolutely Doppler track other probes. Unfortunately, Doppler tracking was not considered for the New Horizons spacecraft, which recently reached Ultima Thule.

\section{CONCLUSION}

This analysis suggests that the relativistic kinetic energy can be explained as the additional energy, necessary to oppose the apparent viscosity of a shear-thickening (dilatant) vacuum. The present investigation also suggests the applicability of the Stokes-Einstein radius to charges which are accelerated through the quantum vacuum (due to electric interaction with the virtual dipoles of the vacuum): this fact too may represent a further step in the understanding of the quantum aspects of relativistic kinetic energy. This research has led indeed to a quantum formula for relativistic kinetic energy, containing a term which is related to quantum potential and vanishes when the formula is applied to accelerated macroscopic bodies: it is here shown that a correct result for the Pioneer anomaly is again obtained. To conclude, considering all the evidences so far collected about vacuum dilatancy, there are reasons to believe - let us repeat - that relativistic kinetic energy does actually correspond to the greater energy necessary to oppose vacuum dilatancy. Relativistic mass would be then the increase of inertia of a body due to the increased viscosity of the dilatant vacuum, leaving mass actually unaffected. The excess energy (with respect to classical kinetic energy) is transferred to the dilatant vacuum until particles collide: in that moment the supplied energy is expressed in the collision, respecting what observed in particle accelerators. Such a viscosity of the vacuum may be due to the Higgs field itself or to diffused dark matter particles in a sea of dark energy. Eventually, the new prospects illustrated in Fig 2 might also open new scenarios for aerospace engineering: for instance, the acceleration of $1 \mathrm{~kg}$ mass to about the speed of light (e.g. $299792457 \mathrm{~m} / \mathrm{s}$ ) would actually require $10^{16} \mathrm{~J}$ instead of $10^{21} \mathrm{~J}$, predicted by special relativity. The greater the mass, the closer the kinetic energy to that of classical physics, being reduced the negative acceleration due to vacuum dilatancy.

\section{ACKNOWLEDGEMENTS}

The author thanks Lorenzo Brandi and the anonymous reviewers for the useful suggestions.
[1] FEDI, M. 2019, Physical vacuum as a dilatant fluid yields exact solutions to the Pioneer anomaly and Mercury's perhelion precession, Can. J. Phys., 97(4): 417-420, doi:10.1139/cjp-2018-0744

[2] TAYLOR, E.F., WHEELER, J.A. 1992, Spacetime Physics, second edition, New York, W.H. Freeman and Company, pp. 248-249

[3] OKUN, L. B. 1989, Physics Today, 42 (6): 31-36
[4] FEDI, M. 2018, Preprint at: https://hal.archives-ouvertes.fr/hal-01863734

[5] Editorial, Nature Physics 8, 635 (2012)

[6] S.G. TURYSHEV, V.T. TOTH, G. KINSELLA, S. Ch. LEE, S.M. LOK and J. ELLIS, 2012, Phys. Rev. Lett. 108:241101

[7] R.O. FIMMEL, J. VAN ALLEN, E. BURGESS, 1980, Pioneer: first to Jupiter, Saturn, and beyond. NASA Scientific and Technical Information Office, Washington D.C., USA

[8] HAWKING, S., MILNER, Y., URL (checked March 21 2019): https://www.theguardian.com/science/2016/apr/12/ stephen-hawking-and-yuri-milner-launch-100m-star-voyage 